\title{
Patterns in Zika Virus Testing and Infection, by Report of Symptoms and Pregnancy Status — United States, January 3-March 5, 2016
}

\begin{abstract}
Sharoda Dasgupta, PhD ${ }^{1,2}$; Sarah Reagan-Steiner, MD³; Dana Goodenough, MPH ${ }^{4}$; Kate Russell, MD 1,5 ; Mary Tanner, MD ${ }^{1,2}$; Lillianne Lewis, MD 1,6 ; Emily E. Petersen, $\mathrm{MD}^{7}$; Ann M. Powers, PhD ${ }^{8}$; Krista Kniss, $\mathrm{MPH}^{5}$; Dana Meaney-Delman, MD 9 ; Titilope Oduyebo, MD ${ }^{1,7}$; Dan O’Leary, DVM ${ }^{10}$; Sophia Chiu, MD ${ }^{1,11}$; Pamela Talley, MD ${ }^{1,12}$; Morgan Hennessey, DVM ${ }^{1,8}$; Susan Hills, MBBS ${ }^{8}$; Amanda Cohn, MD ${ }^{13}$; Christopher Gregory, MD ${ }^{14 ;}$ The Zika Virus Response Epidemiology and Laboratory Team
\end{abstract}

\section{On April 15, 2016 this report was posted as an MMWR Early Release on the MMWR website (http://www.cdc.gov/mmwr).}

CDC recommends Zika virus testing for potentially exposed persons with signs or symptoms consistent with Zika virus disease, and recommends that health care providers offer testing to asymptomatic pregnant women within 12 weeks of exposure. During January 3-March 5, 2016, Zika virus testing was performed for 4,534 persons who traveled to or moved from areas with active Zika virus transmission; 3,335 (73.6\%) were pregnant women. Among persons who received testing, $1,541(34.0 \%)$ reported at least one Zika virus-associated sign or symptom (e.g., fever, rash, arthralgia, or conjunctivitis), $436(9.6 \%)$ reported at least one other clinical sign or symptom only, and 2,557 (56.4\%) reported no signs or symptoms. Among 1,541 persons with one or more Zika virus-associated symptoms who received testing, $182(11.8 \%)$ had confirmed Zika virus infection. Among the 2,557 asymptomatic persons who received testing, 2,425 (94.8\%) were pregnant women, seven $(0.3 \%)$ of whom had confirmed Zika virus infection. Although risk for Zika virus infection might vary based on exposure-related factors (e.g., location and duration of travel), in the current setting in U.S. states, where there is no local transmission, most asymptomatic pregnant women who receive testing do not have Zika virus infection.

Zika virus is a flavivirus primarily transmitted by Aedes species mosquitoes $(1,2)$ that has recently spread in the Region of the Americas (2). From January 1, 2015 to April 13, 2016, a total of 358 travel-associated cases of Zika virus disease were reported from U.S. states, 351 of which were in persons who traveled to or moved from areas with active Zika virus transmission (http://www.cdc.gov/zika/index.html). Most Zika virus infections are asymptomatic or cause mild clinical disease (3). Among persons with clinical illness, signs and symptoms commonly include one or more of the following: fever, rash, arthralgia, and conjunctivitis (3,4). Zika virus infection during pregnancy has been causally linked to congenital microcephaly and has been associated with other adverse pregnancy outcomes, including pregnancy loss $(5-8)$. CDC recommends that persons with possible exposure to Zika virus receive testing if they have symptoms of Zika virus disease within 2 weeks of exposure. On February 12, 2016, CDC recommended that health care providers offer testing to asymptomatic pregnant women with possible exposure to Zika virus (9).

CDC calculated the number of persons in the 50 U.S. states and District of Columbia (DC) who traveled to or moved from areas of active Zika virus transmission and received testing for Zika virus infection in early 2016, and the proportion of tested persons who had evidence of confirmed Zika virus infection or recent unspecified flavivirus infection, by pregnancy status and presence of reported signs and symptoms. This analysis included specimens that were received for Zika virus testing at CDC's Arboviral Diseases Branch during January 3-March 5, 2016, corresponding to epidemiologic weeks 1-9. Confirmed Zika virus infection was defined as detection of 1) Zika virus RNA by reverse transcription-polymerase chain reaction (RT-PCR) or 2) antiZika immunoglobulin M (IgM) antibodies by enzyme-linked immunosorbent assay (ELISA) with neutralizing antibody titers against Zika virus, at levels $\geq 4$-fold higher than those against dengue virus. Recent unspecified flavivirus infection was defined as detection of anti-Zika or anti-dengue virus IgM antibodies by ELISA with $<4$-fold difference in neutralizing antibody titers between Zika and dengue viruses. State and local health departments collected information on clinical signs and symptoms. Zika virus-associated signs and symptoms were defined as at least one of the following: fever, rash, arthralgia, or conjunctivitis (5). Other signs and symptoms not necessarily associated with Zika virus disease were defined as one or more of the following: headache, myalgia, vomiting, diarrhea, edema, oral ulcers, chills, influenza-like illness, or malaise. Persons who reported no symptoms were considered to be asymptomatic. All persons tested in this analysis had traveled to or moved from areas with active Zika virus transmission. Suspected cases of sexually transmitted and congenital Zika virus disease were excluded from the analysis.

During January 3-March 5, 2016, Zika virus testing was performed for 4,534 persons (Table), among whom 3,335 (73.6\%) were pregnant women. Among all persons receiving testing, 197 (4.3\%) had confirmed Zika virus infection, 55 (1.2\%) had recent unspecified flavivirus infection, and 4,282 (94.4\%) had no evidence of recent Zika virus infection. Among all persons receiving testing, $1,541(34.0 \%)$ reported one or more Zika 
virus-associated symptoms, $436(9.6 \%)$ reported at least one other symptom only, and 2,557 (56.4\%) were asymptomatic. Among persons with at least one Zika virus-associated symptom, $620(40.2 \%)$ were pregnant women; among persons with at least one other symptom only, $290(66.5 \%)$ were pregnant women; and among persons with no symptoms, 2,425 (94.8\%) were pregnant women.

During epidemiologic weeks 1-5 (weeks ending January 9February 6, 2016), $<10 \%$ of persons receiving testing were asymptomatic (Figure). After the recommendation to offer serologic testing to asymptomatic pregnant women was published on February 12, 2016 (9), the proportion of persons receiving testing for Zika virus infection who were asymptomatic increased, ranging from $26.1 \%$ to $75.9 \%$ during epidemiologic weeks 6-9. The proportion of persons who received testing who had confirmed Zika virus infection decreased from $33.3 \%$ (epidemiologic week 1) to $1.5 \%$ (week 9).

Among all persons with one or more Zika virus-associated symptoms, $182(11.8 \%)$ had confirmed Zika virus infection and $41(2.7 \%)$ had recent unspecified flavivirus infection (Table). Among persons who reported one or more other symptoms only, eight (1.8\%) had confirmed Zika virus and three $(0.7 \%)$ had recent unspecified flavivirus infection. Among asymptomatic persons, seven $(0.3 \%)$ had confirmed Zika virus and $11(0.4 \%)$ had recent unspecified flavivirus infection.

Among 3,335 pregnant women receiving testing, 28 (0.8\%) had confirmed Zika virus infection and $19(0.6 \%)$ had recent unspecified flavivirus infection. Among pregnant women with at least one Zika virus-associated symptom, 18 (2.9\%) had confirmed Zika virus infection, and nine $(1.5 \%)$ had recent unspecified flavivirus infection. Among 2,425 asymptomatic pregnant women, only seven $(0.3 \%)$ had confirmed Zika virus infection, and $10(0.4 \%)$ had recent unspecified flavivirus infection. Among pregnant women tested after guidelines were expanded to recommend testing of asymptomatic pregnant women (epidemiologic weeks 6-9), seven (35\%) of 20 pregnant women with confirmed Zika virus infection were asymptomatic. Among the seven asymptomatic pregnant women with confirmed Zika virus infection, five were residing in areas with active Zika virus transmission at some time during their pregnancy and two were short-term travelers.

\section{Discussion}

Overall, relatively few persons receiving testing for Zika virus at CDC had confirmed Zika virus infection, and the proportion with confirmed Zika virus infection was higher among persons who reported at least one Zika virus-associated symptom than among persons with other symptoms only or asymptomatic persons. These results reflect the current situation in U.S. states and DC, where there is no local mosquito-borne transmission; results of testing in areas with active Zika virus transmission might be different. Although confirmed Zika virus infection was identified in seven $(0.3 \%)$ asymptomatic pregnant women who received testing, it is reassuring that the proportion of asymptomatic pregnant women with confirmed Zika virus infection in this report was low. However, because

TABLE. Zika virus testing outcomes among persons with specimens tested at CDC's Arboviral Diseases Branch, by Zika virus infection status, reported symptoms, and pregnancy status* - United States, January 3-March 5, $2016^{\dagger}$

\begin{tabular}{|c|c|c|c|c|}
\hline & $\begin{array}{c}\geq 1 \text { Zika virus-associated } \\
\text { symptom }\end{array}$ & $\geq 1$ other symptom onlyq & No symptoms & Total \\
\hline Testing outcome & No. (\%) & No. (\%) & No. (\%) & No. (\%) \\
\hline \multicolumn{5}{|l|}{ All persons tested } \\
\hline Confirmed Zika virus infection & $182(11.8)$ & $8(1.8)$ & $7(0.3)$ & $197(4.3)$ \\
\hline Recent unspecified flavivirus infection & $41(2.7)$ & $3(0.7)$ & $11(0.4)$ & $55(1.2)$ \\
\hline No Zika virus infection & $1,318(85.5)$ & $425(97.5)$ & $2,539(99.3)$ & $4,282(94.4)$ \\
\hline Total & $1,541(100)$ & $436(100)$ & $2,557(100)$ & $4,534(100)$ \\
\hline \multicolumn{5}{|l|}{ Pregnant women* } \\
\hline Confirmed Zika virus infection & $18(2.9)$ & $3(1.0)$ & $7(0.3)$ & $28(0.8)$ \\
\hline Recent unspecified flavivirus infection & $9(1.5)$ & $0(0)$ & $10(0.4)$ & $19(0.6)$ \\
\hline No Zika virus infection & $593(95.7)$ & $287(99.0)$ & $2,408(99.3)$ & $3,288(98.6)$ \\
\hline Total & $620(100)$ & $290(100)$ & $2,425(100)$ & $3,335(100)$ \\
\hline \multicolumn{5}{|c|}{ Other persons (excluding pregnant women) } \\
\hline Confirmed Zika virus infection & $164(17.8)$ & $5(3.4)$ & $0(0)$ & $169(14.1)$ \\
\hline Recent unspecified flavivirus infection & $32(3.5)$ & $3(2.1)$ & $1(0.8)$ & $36(3.0)$ \\
\hline No Zika virus infection & 725 (78.7) & $138(94.5)$ & $131(99.2)$ & $994(82.9)$ \\
\hline Total & $921(100)$ & $146(100)$ & $132(100)$ & $1,199(100)$ \\
\hline
\end{tabular}

* Determined at the time of illness onset (or date of specimen collection, among asymptomatic persons).

${ }^{\dagger}$ As of April 11, 2016.

$\S$ Fever, rash, arthralgia, or conjunctivitis.

" Headache, myalgia, vomiting, diarrhea, edema, oral ulcers, chills, influenza-like illness, or malaise. 
FIGURE. Number of symptomatic and asymptomatic persons who received Zika virus testing, ${ }^{*}$ by pregnancy status, and percentage of positive results among all persons tested, persons with symptoms, and asymptomatic persons, by epidemiologic week - United States, January 3March 5, 2016 ${ }^{\dagger}$

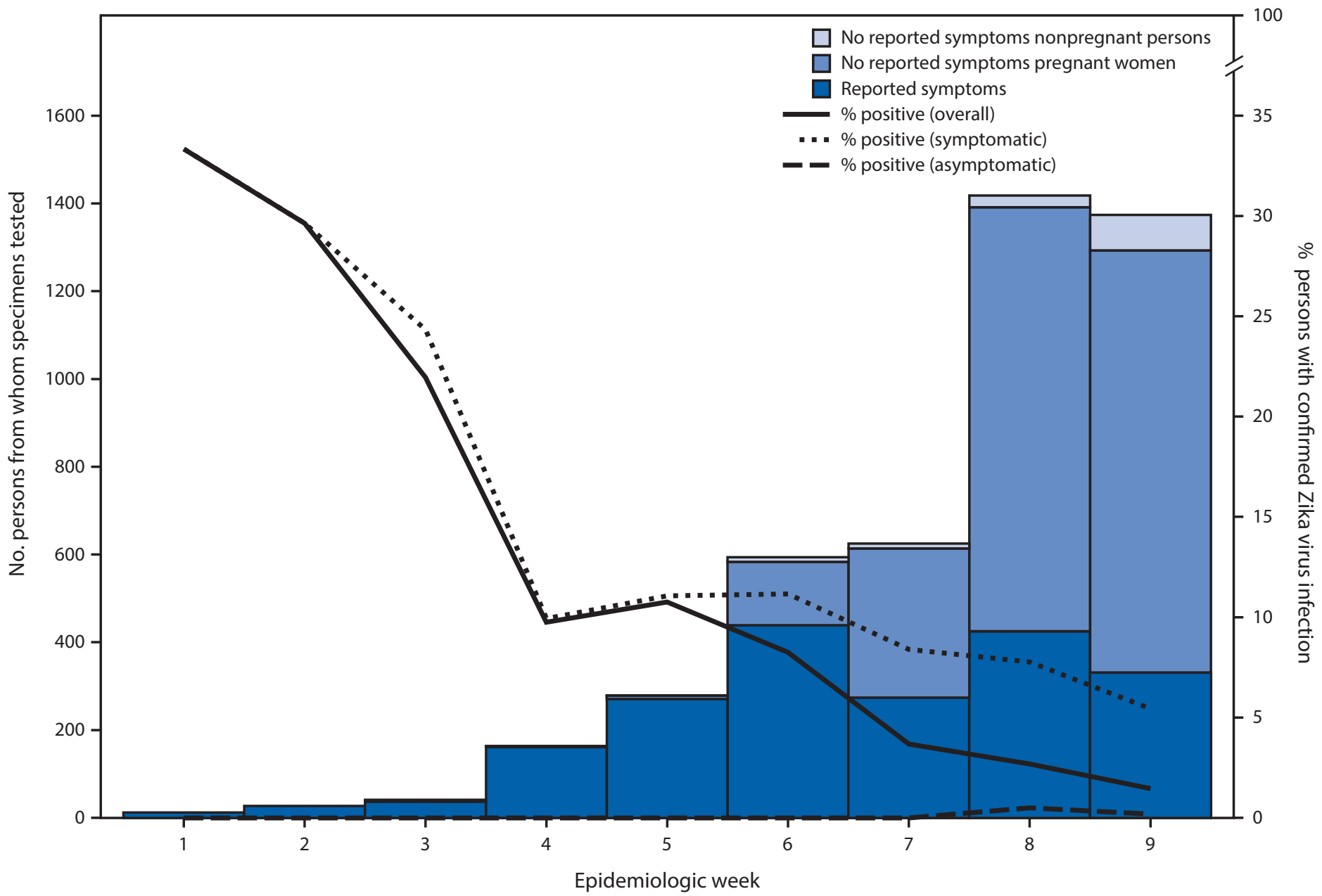

* Testing performed at CDC's Arboviral Diseases Branch.

${ }^{\dagger}$ As of April 11, 2016.

of the potential serious adverse pregnancy and neonatal outcomes associated with maternal Zika virus infection, health care providers should continue to offer testing to pregnant women with potential exposure to Zika virus, even if they do not have symptoms (9). Follow-up of women with confirmed Zika virus infection or recent unspecified flavivirus infection during pregnancy is important to identify congenital Zika virus infection and other possible adverse pregnancy outcomes.

The findings in this report are subject to at least five limitations. First, because testing might have been performed weeks after potential exposure, persons might not recall symptoms, particularly if they were mild. Second, only tests performed at CDC's Arboviral Diseases Branch were included in this analysis. Some state health departments were testing for Zika virus during this time and the total number of cases reported in this period from U.S. states (http://www.cdc.gov/zika/index.html) exceeds the number of cases described in this analysis. Third, this study did not account for heterogeneous exposure risk among persons receiving testing. Travel-associated exposure can vary by location, duration, accommodations, and activities during travel. Fourth, findings in this report are not generalizable to residents of areas with active Zika virus transmission. Finally, patients with unspecified flavivirus infection likely experienced a previous infection with or had been vaccinated against other related flaviviruses making results difficult to interpret. In the setting of the current Zika virus outbreak and because of the concern for adverse fetal effects, pregnant women with unspecified flavivirus infection should follow CDC guidance for pregnant women with possible Zika virus infection (10).

In the U.S. states and DC, the proportion of persons who traveled to or moved from areas with active Zika virus 


\section{Summary}

What is already known about this topic?

Zika virus is an emerging mosquito-borne flavivirus. Travelassociated cases of Zika virus disease have been reported in the United States. Zika virus infection during pregnancy has been causally linked to congenital microcephaly and has been associated with other adverse pregnancy outcomes, including pregnancy loss. On February 12, 2016, CDC recommended that health care providers offer testing for Zika virus disease to asymptomatic pregnant women with possible exposure to Zika virus.

What is added by this report?

During January 3-March 5, 2016, Zika virus testing was performed for 4,534 persons from the U.S. states and District of Columbia (DC), among whom 3,335 (73.6\%) were pregnant women. Among 1,541 persons with one or more Zika-virus associated symptoms who received testing and reported symptoms, $182(11.8 \%)$ had confirmed Zika virus infection. Only seven $(0.3 \%)$ of 2,425 asymptomatic pregnant women who received testing had confirmed Zika virus infection.

What are the implications for public health practice?

Among persons from U.S. states and DC receiving testing for Zika virus, few persons had confirmed Zika virus infection. Approximately $99 \%$ of asymptomatic pregnant women who received testing did not have Zika virus infection. In the current U.S. setting, where most exposure is travel-associated, the likelihood of Zika virus infection among asymptomatic persons is low. Given the potential for adverse pregnancy and infant outcomes associated with Zika virus infection, health care providers should continue to offer Zika virus testing to asymptomatic pregnant women with potential exposure.

transmission, who received testing, and who had confirmed Zika virus infection was substantially higher in symptomatic than asymptomatic persons. Furthermore, 64\% of pregnant women with confirmed Zika virus infection had at least one Zika virus-associated symptom, and approximately $99 \%$ of asymptomatic pregnant women who received testing did not have Zika virus infection. Because of the potential for adverse outcomes associated with Zika virus infection during pregnancy and the lack of current understanding of the risks for infection in asymptomatic pregnant women, health care providers should continue to offer Zika virus testing to asymptomatic pregnant women with potential exposure (9). Although individual risk for Zika virus infection will differ on the basis of exposure, these data suggest that in the current setting in U.S. states, where most exposure is travel-associated, most asymptomatic persons do not have Zika virus infection.
${ }^{1}$ Epidemic Intelligence Service, CDC; ${ }^{2}$ Division of HIV/AIDS Prevention, National Center for HIV/AIDS, Viral Hepatitis, STD, and TB Prevention, CDC; ${ }^{3}$ Immunization Services Division, National Center for Immunization and Respiratory Diseases, CDC; ${ }^{4} \mathrm{Oak}$ Ridge Institute for Science and Education (ORISE) Fellow, Division of Human Development and Disability, National Center on Birth Defects and Developmental Disabilities, CDC; 5 Influenza Division, National Center for Immunization and Respiratory Diseases, CDC; ${ }^{6}$ National Center for Environmental Health, Division of Environmental Hazards and Health Effects, CDC; ${ }^{7}$ Division of Reproductive Health, National Center for Chronic Disease Prevention and Health Promotion, CDC; ${ }^{8}$ Division of Vector-Borne Diseases, National Center for Emerging and Zoonotic Infectious Diseases, CDC; ${ }^{9}$ Office of the Director, National Center for Emerging and Zoonotic Infectious Diseases, CDC; ${ }^{10}$ Division of State and Local Readiness, Office of Public Health Preparedness and Response, CDC;

${ }^{11}$ National Institute for Occupational Safety and Health, Division of Surveillance, Hazard Evaluations, and Field Studies; ${ }^{12}$ Minnesota Department of Health; ${ }^{13}$ Office of the Director, National Center for Immunization and Respiratory Diseases, CDC; ${ }^{14}$ Division of Global Health Protection, Center for Global Health, CDC.

Corresponding author: Sarah Reagan-Steiner, sor1@cdc.gov, 404-639-8205.

\section{The Zika Virus Response Epidemiology and Laboratory Team}

Janeen Laven, Olga Kosoy, Amanda Panella, Marc Fischer, Amanda Calvert, Jane Basile, Christin Goodman, Robert Lanciotti, Jeremy Ledermann, Jennifer Lehman, Eric Mossel, Katherine Chu, Charles Futoran, Paul Burns, Sandor Karpathy, Joseph Singleton, Aubree Kelly, Michelle Allerdice, Rachael Priestley, Kelly Fitzpatrick, Sher'i Brooks, Avi Stein, Molly Lauterbach, Ryan Pappert, Adam Replogle, Brook Yockey, Chris Sexton, John Young (all these individuals meet collaborator criteria).

\section{References}

1. Hayes EB. Zika virus outside Africa. Emerg Infect Dis 2009;15:1347-50. http://dx.doi.org/10.3201/eid1509.090442

2. CDC. Areas with Zika. Atlanta, GA: US Department of Health and Human Services, CDC; 2016. http://www.cdc.gov/zika/geo/

3. Duffy MR, Chen TH, Hancock WT, et al. Zika virus outbreak on Yap Island, Federated States of Micronesia. N Engl J Med 2009;360:2536 43. http://dx.doi.org/10.1056/NEJMoa0805715

4. Armstrong P, Hennessey M, Adams M, et al. Travel-associated Zika virus disease cases among US residents-United States, January 2015February 2016. MMWR Morb Mortal Wkly Rep 2016;65:286-9. http://dx.doi.org/10.15585/mmwr.mm6511e1

5. Oliveira Melo AS, Malinger G, Ximenes R, Szejnfeld PO, Alves Sampaio S, Bispo de Filippis AM. Zika virus intrauterine infection causes fetal brain abnormality and microcephaly: tip of the iceberg? Ultrasound Obstet Gynecol 2016;47:6-7. http://dx.doi.org/10.1002/uog.15831

6. Martines RB, Bhatnagar J, Keating MK, et al. Notes from the field: evidence of Zika virus infection in brain and placental tissues from two congenitally infected newborns and two fetal losses-Brazil, 2015. MMWR Morb Mortal Wkly Rep 2016;65:159-60. http://dx.doi.org/10.15585/mmwr.mm6506e1

7. Mlakar J, Korva M, Tul N, et al. Zika virus associated with microcephaly. N Engl J Med 2016;374:951-8. http://dx.doi.org/10.1056/ NEJMoa1600651 
8. Rasmussen SA, Jamieson DJ, Honein MA, Petersen LR. Zika virus and birth defects—-reviewing the evidence for causality. N Engl J Med 2016. E-pub April 13, 2016. http://dx.doi.org/10.1056/NEJMsr1604338

9. Oduyebo T, Petersen EE, Rasmussen SA, et al. Update: interim guidelines for health care providers caring for pregnant women and women of reproductive age with possible Zika virus exposure-United States, 2016. MMWR Morb Mortal Wkly Rep 2016;65:122-7. http://dx.doi. org/10.15585/mmwr.mm6505e2
10. Petersen EE, Polen KN, Meaney-Delman D, et al. Update: interim guidance for health care providers caring for women of reproductive age with possible Zika virus exposure-United States, 2016. MMWR Morb Mortal Wkly Rep 2016;65:315-22. http://dx.doi.org/10.15585/mmwr.mm6512e2 